\title{
An Efficient Method to Measure Evidence Conflict
}

\author{
Baojie Liu ${ }^{1, *}$, Qingwen Yang ${ }^{1}$, Xiang $\mathrm{Wu}^{1}$, Yujuan Guo ${ }^{2}$ and Shidong Fang ${ }^{1}$ \\ ${ }^{1}$ Fifth Department, Army Officer Academy of PLA, 451 Huangshan Road, Hefei, 230031, China \\ ${ }^{2}$ University Office, Anhui Radio \& TV University, 398 Tongcheng Rd, Hefei, 230022, China \\ ${ }^{*}$ Corresponding author
}

\begin{abstract}
Dempster Shafer evidence theory, as an uncertain information fusion technology, is widely used in various fields of information fusion. However, when there is a highly conflict between two pieces of evidence, counterintuitive results are obtained by classical Dempster's combination rule. Therefore, it is very important to measure the conflict between two pieces of evidence. Based on the analysis of some typical conflict measurement methods, a new model to represent conflict was constructed with the Euclidean distance function and the Jaccard similarity coefficient. Some numerical examples illustrate that the proposed method can measure the degree of conflict between the two pieces of evidence and overcome the shortcomings of the classical conflictive coefficient, Jousselme evidence distance and Pignistic probability distance to a certain extent.
\end{abstract}

Keywords-D-S evidence theory; evidence conflict; jaccard similarity coefficient ; conflict measure; information fusion

\section{INTRODUCTION}

As a method of uncertainty reasoning, Dempster-Shafer (D-S) evidence theory[1,2] can model and reason in uncertain problems and realize the fusion of no a priori information, and has been widely used in target recognition tracking, fault diagnosis, network security assessment and other areas[3-9]. However, when the evidence is highly conflicting, the results of the fusion of evidence often appear contrary to the facts. To solve this problem, domestic and foreign scholars have carried on the analysis and explored, and many improvement methods have been proposed, to a certain extent, a lack of evidence theory has been made up. On the whole, the existing improved algorithms are divided into two main categories: First, by modifying the combination rule of D-S evidence theory, the improved algorithm realizes the redistribution of conflicting evidence[10-12]. The reference[10] introduces the new elements into the identification framework which considers that all conflicts can not provide useful information, and all conflict information is allocated to new elements. The reference[11] introduces the credibility of the evidence and assigns the conflicting information to all the coke sets of evidence. The main content of this kind of method research is what proposition should be assigned to conflict and what proportion should the conflict be distributed. The main content of this kind of methods is that the conflict should be allocated to what proposition and the conflict should be allocated according to what proportion. Second, the conflict evidence is pre-modified before fusion, and then, according to the combination rule of D-S evidence theory, conflict evidence is merged[13-18]. In the reference[13], the basic probability assignment (BPA) is arithmetically averaged, and the resulting evidence is used to replace the original n-evidence. In the reference[14], the concept of confidence factor is introduced. The reliability coefficient is used to revise the evidence of conflict. In the reference[15], the Jousselme distance function is introduced to measure the conflict degree of evidence. On this basis, the trust coefficient is defined to modify the evidence of conflict. In the reference[16-18], the new method of improving the weight of evidence is proposed, and the results are very good. The main content of this kind of methods is to use what method to pre process the conflict evidence, which makes the conflict evidence fusion better.

However, in the actual application process, there are many reasons causing the conflict of evidence source, such as sensor characteristic differences, sensor performance and evidence description of different subjects, people's cognition of things incomplete may lead to the change of BPA, Therefore, it is inappropriate to blame the reason of the paradox in the rules of evidence combination, Based on the above reasons, this paper clearly followed the second kinds of research methods, before choosing the appropriate method to improve the modified conflict evidence to determine the degree of conflict between two pieces of evidence is a crucial problem. In order to better describe the degree of conflict between two pieces of evidence, the paper presents a method to characterize the conflict between two pieces of evidence based on the Euclidean distance and the Jaccard similarity coefficient. This method can be used to determine whether there is a conflict between two pieces of evidence, in order to determine whether the use of D$\mathrm{S}$ combination rules or other synthetic methods.

\section{D-S EVIDENCE THEORY AND ITS EXISTING PROBLEMS}

This section simply introduces the basic principle of D-S evidence theory, the detailed information can be referenced in the literature[18].

Definition 1: set $\Omega$ as the sample space, $2^{\Omega}$ is the power set of $\Omega$, if the function $m: 2^{\Omega} \rightarrow[0,1]$ satisfies: $m(\varnothing)=0, \sum_{A \subseteq \Omega} m(A)=1$, then $m$ is the basic confidence distribution function on $2^{\Omega}$, and $m(A)$ is called the basic credibility of evidence against $A$. If $\forall A \subset \Omega$ and $m(A)>0$, then $A$ is the focal element of evidence.

Definition 2: $m_{1}, m_{2} \cdots, m_{n}$ is the basic confidence distribution function, then the D-S evidence theory synthesis rule is defined[1,2]: 


$$
\left\{\begin{array}{cc}
m(A)=0 & A=\varnothing \\
m(A)=\frac{1}{1-K} \sum_{\cap A_{i}=A} \prod_{1 \leq i \leq n} m_{i}\left(A_{i}\right) & A \neq \varnothing
\end{array}\right.
$$

where $K=\sum_{\cap A_{i}=\varnothing} \prod_{1 \leq i \leq n} m_{i}\left(A_{i}\right)$ is the conflict coefficient, which indicates the degree of conflict between two pieces of evidence. The greater the value of $K$ indicates the greater the conflict between two pieces of evidence.

In the Dempster combination rule, the conflict coefficient $K \in[0,1]$. If $K=1$, Dempster combination rules can not be calculated; If $K \rightarrow 1$, when using Dempster combination rules to merge the evidence of high conflict, the results will appear inconsistent with the actual or the appearance of consistent evidence "miscarriage of justice" and other issues.

Example 1 Putative recognition framework $\Omega=\{a, b, c\}$, the system has two evidences as follows:

Evidence 1: $m_{1}\{a\}=0.99, m_{1}\{b\}=0.01$;

Evidence 2: $m_{2}\{c\}=0.99, m_{2}\{b\}=0.01$.

Using Dempster combination rule to synthesize two evidences, the calculation results are as follows: $K=0.9999, m\{a\}=m\{c\}=0, m\{b\}=1$. It can be seen that the evidence 1,2 is highly conflicting, the results is contrary to common sense, because $m_{1}$ and $m_{2}$ on the $b$ support rate is very low, but the combination results have the greatest degree of confidence.

Many scholars have studied in this respect, and have come to a more consistent conclusion, that is, to resolve the conflict of evidence fusion, then there should be the first to determine whether there is a conflict between the evidence? How to quantitatively describe the extent of the conflict of evidence? It is proved that the conflict coefficient in D-S evidence theory can not effectively measure the conflict between evidences. As can be seen from the following example.

Example 2 Putative recognition framework $\Omega=\{a, b, c, d, e\}$, the system has two evidences as follows:

Evidence 1: $m_{1}\{a\}=m_{1}\{b\}=m_{1}\{c\}=m_{1}\{d\}=m_{1}\{e\}=0.2$

Evidence2 $m_{2}\{a\}=m_{2}\{b\}=m_{2}\{c\}=m_{2}\{d\}=m_{2}\{e\}=0.2$

The calculation result of conflict coefficient is $K=0.8$. According to the classical D-S evidence theory, it can be concluded that the two pieces of evidence are highly conflicting. But in fact, there is no conflict between two pieces of evidence, which is two identical evidence. It can be seen that the conflict coefficient in the D-S theory can not be used to characterize the conflict between two pieces of evidence. What variable should be used to characterize the conflict between the evidence?

\section{AnAlysis Of EVIDENCE CONFLICT METHOD}

Since the conflict coefficient in the D-S theory can not be used to characterize the conflict between two pieces of evidence, what variables should be used to represent the conflict between two pieces of evidence? At present, there are many methods to measure the conflict of evidence, for example, Jousselme distance function[14], pignistic probability distance[19], information entropy definition correlation coefficient[20], etc, which are used to measure the degree of conflict between two pieces of evidence, the following some numerical examples are given to analyze the existing methods of conflict measurement.

\section{A. Jousselme Evidence Distance}

In 2001, Jousselme published an article devoted to the analysis of the distance between evidences[21], which introduced the distance of evidence to measure the size of the conflict between two pieces of evidence.

Definition 3 Identification framework $\Omega$ contains $N$ different proposition, $E_{p(\Omega)}$ is the space of all subsets generated $\Omega$. A basic probability assignment BPA is a vector $\vec{m}_{i}$ with a coordinate system of $m\left(A_{i}\right)$ in $E_{p(\Omega)}$, and the following conditions are satisfied:

$$
\left\{\begin{array}{l}
\sum_{i=1}^{2^{N}} m\left(A_{i}\right)=1, \quad m\left(A_{i}\right) \geq 0 \\
i=1, \cdots, 2^{N} ; A_{i} \in P(\Omega)
\end{array} .\right.
$$

Definition 4 In the identification framework, the Jousselme distance of BPA function of two pieces of evidence is:

$$
d_{B P A}\left(m_{1}, m_{2}\right)=\sqrt{1 / 2\left(\vec{m}_{1}-\vec{m}_{2}\right)^{T} \underline{\underline{D}}\left(\vec{m}_{1}-\vec{m}_{2}\right)},
$$

where: $\underline{D}$ is a $2^{N} \times 2^{N}$ matrix, the elements in the matrix are:

$$
D(A, B)=\frac{|A \cap B|}{|A \cup B|}, A, B \in P(\Omega) .
$$

Distance function $d_{B P A}\left(m_{1}, m_{2}\right)$ can be used to measure the degree of conflict and differences between the two pieces of evidence. The greater the value of $d_{B P A}\left(m_{1}, m_{2}\right)$ indicates the greater the conflict between two pieces of evidence. Which solves the problem that the conflict coefficient in the classical D-S evidence theory has miscarriage of justice in the case of consistent evidence, but the algorithm has high complexity, complicated calculation process and some problems.

Example 3 Putative recognition framework $\Omega=\{a, b, c, d, e\}$, the system has three evidences as follows:

Evidence 1: $m_{1}\{a\}=0.5, m_{1}\{b\}=0.3, m_{1}\{c\}=0.2$; 
Evidence 2: $m_{2}\{a\}=0.2, m_{2}\{b\}=0.3, m_{2}\{c\}=0.5$;

Evidence 3: $m_{3}\{a\}=0, m_{3}\{b\}=0.1, m_{3}\{c\}=0.9$.

According to the evidence distance formula, the following results can be calculated: $d_{B P A}\left(m_{1}, m_{2}\right)=0.3, d_{B P A}\left(m_{1}, m_{2}\right)=0.35$. It can be seen from the results that the conflict between evidence 2 and evidence 3 is greater than the conflict between evidence 1 and evidence 2, but it can be seen from the intuition that evidence 1 supports $a, 2$ and 3 support $c$. The conflict between evidence 2 and evidence 3 is greater than the conflict between evidence 1 and evidence 2, Thus, the conflict between evidence 1 and evidence 2 should be greater than the conflict between evidence 2 and evidence 3 , which is inconsistent with the results obtained from the evidence distance. This is because the matrix is a diagonal matrix, each evidence in each focal element are independent of each other, in a conflict measure, it amplifies the larger focal element difference, namely distance of evidence can not correctly reflect the differences between two pieces of evidence.

\section{B. Pignistic Probability Distance}

In 2006 Liu[19] introduced the Pignistic probability distance.

Definition 5 Let $m$ be the BPA function under the frame $\Omega$ and $\omega$ is the subproposition of the proposition $A$, then the Pignistic probability function is:

$$
\begin{gathered}
\operatorname{Bet}_{m}(A)=\sum_{\omega \subseteq A} \operatorname{BetP}_{m}(\omega), \\
\left\{\begin{array}{c}
\operatorname{Bet}_{m}(\omega)=\sum_{A \subseteq \Omega, \omega \subseteq A} \frac{1}{|A|} \frac{m(A)}{1-m(\varnothing)}, \\
m(\varnothing)=1, \Omega \rightarrow[0,1]
\end{array}\right.
\end{gathered}
$$

where $|A|$ is the potential of subset $A$.

By using the $\operatorname{BetP}_{m}$ function, the distance between two pieces of evidences $\operatorname{difBet} P_{m}$ can be defined.

Definition 6 The two evidence $m_{1}$ and $m_{2}$ are BPA functions on the recognition frame $\Omega$, then the Pignistic distance between the two pieces of evidence is:

$$
\operatorname{difBetP} P_{m_{1}}^{m_{2}}=\max _{A \subseteq \Omega}\left(\left|\operatorname{BetP}_{m_{1}}(A)-\operatorname{BetP}_{m_{2}}(A)\right|\right)
$$

Pignistic probability distance is the maximum value of the difference between the A functions. The maximum difference between different evidence points is described, and the conflict of evidence can be described effectively, but there are some shortcomings.
Example 4 Putative recognition framework $\Omega=\{a, b, c\}$, the system has two evidences as follows:

Evidence 1: $m_{1}\{a\}=0.7, m_{1}\{b\}=0.15, m_{1}\{c\}=0.15$;

Evidence 2: $m_{2}\{a\}=0.15, m_{2}\{b\}=0.7, m_{2}\{c\}=0.15$.

$\operatorname{difBet} P_{m_{1}}^{m_{2}}=0.55$ are given by the Pignistic probability distance formula, which shows that there is little conflict between the two evidences and the evidence, but in fact $m_{1}$ supports $a$ and $m_{2}$ supports $b$, that is, they support different coke elements, and the evidence conflicts should be compared large, which is slightly different from the actual situation. Although the Pignistic probability distance can be used to characterize the conflict size, there is still room for further improvement.

On the basis of several commonly used evidence conflict measurement methods, many improved algorithms are proposed. In [22], a new conflict of evidence approach is proposed to reflect the non-inclusiveness and divergence of evidence, using the conflict coefficient and the Jousselme evidence distance. In [23], the size of the conflict is represented by the geometric mean of the conflict coefficient and Jousselme's evidence distance. In [19], the conflict coefficient is used to determine whether there is conflict between the two factors and whether the evidence is highly conflicting. In [24], based on the Pignistic probability function, the concept of Tanimoto measure is introduced, and the evidence similarity measure is presented to represent the conflict degree of evidence. In [25], the size of the conflict is represented by the sum of the squares of the conflict coefficient and Jousselme's evidence distance. Based on a brief analysis of the above methods, we propose an improved evidence distance measurement method to measure the conflict size of the existing conflict measurement methods.

\section{AN IMPROVED Method to MEASURE THE CONFLICT OF EVIDENCE}

Based on the Euclidean distance function and Jaccard's similarity coefficient, this paper presents a new method to characterize the degree of evidence conflict.

Definition 7 There are two sets $X$ and $Y$, the proportion of the intersection elements of $X$ and $Y$ in the union of $\mathrm{A}$ and $\mathrm{B}$, known as the two sets of Jaccard similarity coefficients, denoted by the symbol $J(X, Y)$.

$$
J(X, Y)=\frac{|X \cap Y|}{|X \cup Y|} .
$$

If set $\mathrm{X}$ and $\mathrm{Y}$ are empty sets, define $J(X, Y)=1$, $0 \leq J(X, Y) \leq 1 . J(X, Y)$ is a measure of the similarity of two sets of an indicator, the greater the value of $J(X, Y)$ indicates the greater the similarity of the two samples. 
There are two evidences that $m_{1}$ and $m_{2}$ are the BPA functions on the recognition framework $\Omega$, B and $N$ are two $n$ dimensional vectors, and the values of all dimensions are 0 or 1. For example: $m_{1}(0111)$ and $m_{2}(1011)$. We regard the sample as a collection, 1 indicates that the set contains the element, and 0 indicates that the set does not contain the element. Definition: the 1 ;

$p$ : Sample $m_{1}$ and $m_{2}$ are the number of dimensions of

$q$ : Samples A and B are the number of dimensions of 1 and 0 , respectively;

$r$ : Samples A and B are the number of dimensions of 0 and 1 , respectively; 0 ;

$s$ : Sample $m_{1}$ and $m_{2}$ are the number of dimensions of the

Then the similarity coefficient of $m_{1}$ and $m_{2}$ can be expressed as:

$$
J\left(m_{1}, m_{2}\right)=\frac{p}{p+q+r} .
$$

In order to comprehensively consider the inclusion and diversity of evidence, the evidence conflict measure coefficient(ECMC) is defined to characterize the conflict between two pieces of evidence based on the Euclidean distance function and Jaccard's similarity coefficient.

Definition 8 Identification framework $\Omega$ contains $N$ different proposition, $E_{p(\Omega)}$ is the space of all subsets generated $\Omega$. A basic probability assignment BPA is a vector $\vec{m}_{i}$ and $\vec{m}_{j}$ with a coordinate system of $m\left(A_{i}\right)$ and $m\left(A_{j}\right)$ in $E_{p(\Omega)}$, Then the evidence conflict measure coefficient(ECMC) is defined as:

$$
d_{i j}=\sqrt{J\left(\vec{m}_{i}-\vec{m}_{j}\right)\left(\vec{m}_{i}-\vec{m}_{j}\right)^{T}}
$$

If $\left|\vec{m}_{i}-\vec{m}_{j}\right|>1, d_{i j}=1$ is defined. $d_{i j}$ is a measure indicator of the conflict of the two sets, the greater the value of $d_{i j}$ indicates the greater the conflict of the two samples.

In the following example, an example is given to show that the evidence conflict measure coefficient(ECMC) can be used to characterize the conflict between two pieces of evidence. For example 1 mentioned above, using the formula (10) to calculate, the calculation process is as follows

$$
m_{1}=\left\{\begin{array}{ccc}
m(a) & m(b) & m(c) \\
0.99 & 0.01 & 0
\end{array}\right\}, m_{2}=\left\{\begin{array}{ccc}
m(a) & m(b) & m(c) \\
0 & 0.01 & 0.99
\end{array}\right\} .
$$

First, the data are preprocessed

$$
\vec{m}_{1}=\left[\begin{array}{lll}
0.99 & 0.01 & 0
\end{array}\right], \vec{m}_{2}=\left[\begin{array}{lll}
0 & 0.01 & 0.99
\end{array}\right]
$$

All the dimensions of vector $\vec{m}_{1}$ and $\vec{m}_{2}$ are 0 or 1 , and the parameters $\vec{X}$ and $\vec{Y}$ of the Jaccard similarity coefficients are respectively:

$$
\vec{X}=\left[\begin{array}{lll}
1 & 1 & 0
\end{array}\right], \vec{Y}=\left[\begin{array}{lll}
0 & 1 & 1
\end{array}\right]
$$

Then, the ECMC is calculated for the processed data, and the result is as follows:

$$
\begin{aligned}
& J(X, Y)=0.333, \vec{m}_{1}-\vec{m}_{2}=\left[\begin{array}{lll}
0.99 & 0 & -0.99
\end{array}\right] \\
& d_{12}=\sqrt{J\left(\vec{m}_{1}-\vec{m}_{2}\right)\left(\vec{m}_{1}-\vec{m}_{2}\right)^{T}}=\sqrt{0.3333 \times 1.9602}=0.8083
\end{aligned}
$$

From the common sense, two pieces of evidence on the same target support have obvious differences, evidence 1 to support the objectives of the $a$ is 0.99 , and the evidence 2 supports the objectives of the $c$ is 0.99 , which shows that there is a conflict between two pieces of evidence, but the two pieces of evidence also supports $b$. Therefore, it is not possible to rule out the possibility of the "error" caused by the sensor fault, and the results are in line with the actual analysis.

For example 2, the calculated ECMC is 0, indicating that there is no conflict between two pieces of evidence, and the actual understanding is the same; for example 3 , evidence 1 and evidence 2 of the ECMC is $d_{12}=0.424$, the ECMC of evidence 2 and evidence 3 is $d_{23}=0.4$, evidence 1 supports $a$, evidence 2 and Evidence 3 Supports $c$. Therefore, the conflict between evidence 1 and evidence 2 should be greater than the conflict between evidence 2 and evidence 3 , which is consistent with the actual analysis results. According to the formula (3), the Jousselme evidence distance is calculated as follows: $d_{B P A}\left(m_{1}, m_{2}\right)=0.3, d_{B P A}\left(m_{1}, m_{2}\right)=0.35$, the calculation result is not consistent with the fact. For example 4, the ECMC of evidence 1 and 2 is $d_{12}=0.778$ according to formula (10), and the Pignistic probability distance is $\operatorname{difBet} P_{m_{1}}^{m_{2}}=0.55$ according to formula (7), In fact $m_{1}$ supports $a, m_{2}$ supports $b$, that they support the focal element is different, the conflict between the evidence should be relatively large. It can be seen from the above example that the ECMC can overcome the problem that conflict coefficient in the classic DS evidence theory and Jousselme evidence distance can not effectively represent the conflict size between the two pieces of evidences. In measuring conflict between two pieces of evidences, the ECMC is better than the Pignistic probability Distance.

\section{EXAMPLE ANALYSIS}

It has been proved that the conflict coefficient of the D-S evidence theory can not represent the conflict between the evidence. Here are a few examples to further illustrate the ECMC can be used to measure the conflict between the evidence. When the two pieces of evidence are identical, then the use of the above formula to obtain ECMC is 0, indicating that the two pieces of evidence is consistent, there is no conflict between the evidence, support for the same goal is the 
completion of the same. When the evidence is not exactly the same, the ECMC can also represent the conflict between the evidence. The following example illustrates the effectiveness of the ECMC in judging evidence conflicts.

Example 5 Putative recognition framework $\Omega=\{a, b, c\}$, the system has two evidences as follows:

Evidence 1: $m_{1}\{a\}=0.8, m_{1}\{b\}=0.1, m_{1}\{c\}=0.1$;

Evidence 1: $m_{2}\{a\}=0.2, m_{2}\{b\}=0.8, m_{2}\{c\}=0$.

By calculation, the ECMC is 0.757

Example 6 Putative recognition framework $\Omega=\{a, b, c\}$, the system has two evidences as follows:

Evidence 1: $m_{1}\{a\}=0.8, m_{1}\{b\}=0.1, m_{1}\{c\}=0.1$;

Evidence 2: $m_{2}\{a\}=0.8, m_{2}\{b\}=0.2, m_{2}\{c\}=0$.

By calculation, the ECMC is 0.115

From Example 5 and Example 6, it can be seen that there is a high degree of conflict between the evidence when the ECMC is large, and there is no conflict or a little conflict between the evidences when the ECMC is small.

When there is a conflict between the evidence, whether the conflict measure coefficient can reflect the conflict between the evidence is more serious? The following examples are discussed.

Example 7 Putative recognition framework $\Omega=\{a, b\}$, the system has two evidences as follows:

Evidence 1: $m_{1}\{a\}=0.7, m_{1}\{b\}=0.3$;

Evidence 2: $m_{2}\{a\}=0.3, m_{2}\{b\}=0.7$;

By calculation, the ECMC is 0.566

Example 8 Putative recognition framework $\Omega=\{a, b\}$, the system has two evidences as follows:

Evidence 1: $m_{1}\{a\}=0.8, m_{1}\{b\}=0.2$;

Evidence 2: $m_{2}\{a\}=0.2, m_{2}\{b\}=0.8$;

By calculation, the ECMC is 0.849

From the results of Example 7 and Example 8, it can be seen that the ECMC of Example 8 is larger than that of Example 7. The conflict between the two evidences in Example 8 is more obvious through visual analysis. It can be seen that the ECMC can be used to judge whether there is conflict between two evidence and the degree of conflict.

From the above examples have seen this factor of ECMC can characterize the conflict between the evidence, this paper will use the examples in [27] to illustrate the ECMC as the pignistic probability distance proposed by Liu to characterize the conflict between the evidence. the next step for the selection of evidence combination method suitable to lay a good foundation, which can be the next step to select the appropriate evidence synthesis method to lay the foundation.

Example 9 In [27], recognition framework $\Omega=\{1,2,3 \cdots, 20\}$, there are two BPA as follows:

Evidence 1: $m_{1}(2,3,4)=0.05, m_{1}(7)=0.05, m_{1}(\Omega)=0.1$, $m_{1}(A)=0.8$;

Evidence 2: $m_{2}(1,2,3,4,5)=1$.

Where A varies in accordance with $\{1\},\{1,2\}$, $\{1,2,3\}, \cdots,\{1,2,3 \cdots, 20\}$

Table 1 gives the comparison of ECMC to conflict coefficient of the classical D-S evidence theory( $K$ ), Jousselme evidence distance $\left(d_{B P A}\right)$, and pignistic probability distance $\left(\operatorname{difBet} P_{m}\right)$ in 20 cases when $A$ changes.

TABLE I. COMPARISON OF NEW PROPOSED CONFLICT COEFFICIENT WITH CLASSICAL CONFLICT COEFFICIENT

\begin{tabular}{lllll}
\hline & \multicolumn{4}{c}{ Conflict measure } \\
\cline { 2 - 5 } A & $K$ & $d B P A$ & difBetPm & dij \\
\hline$\{1\}$ & 0.05 & 0.814 & 0.730 & 0.819 \\
$\{1,2\}$ & 0.05 & 0.712 & 0.552 & 0.709 \\
$\{1,2,3\}$ & 0.05 & 0.596 & 0.373 & 0.589 \\
$\{1,2, \ldots, 4\}$ & 0.05 & 0.405 & 0.195 & 0.398 \\
$\{1,2, \ldots, 5\}$ & 0.15 & 0.150 & 0.125 & 0.120 \\
$\{1,2, \ldots, 6\}$ & 0.15 & 0.397 & 0.258 & 0.387 \\
$\{1,2, \ldots, 7\}$ & 0.15 & 0.514 & 0.354 & 0.496 \\
$\{1,2, \ldots, 8\}$ & 0.15 & 0.586 & 0.425 & 0.575 \\
$\{1,2, \ldots, 9\}$ & 0.15 & 0.632 & 0.480 & 0.621 \\
$\{1,2, \ldots, 10\}$ & 0.15 & 0.673 & 0.525 & 0.664 \\
$\{1,2, \ldots, 11\}$ & 0.15 & 0.698 & 0.561 & 0.682 \\
$\{1,2, \ldots, 12\}$ & 0.15 & 0.714 & 0.592 & 0.708 \\
$\{1,2, \ldots, 13\}$ & 0.15 & 0.727 & 0.617 & 0.719 \\
$\{1,2, \ldots, 14\}$ & 0.15 & 0.739 & 0.639 & 0.728 \\
$\{1,2, \ldots, 15\}$ & 0.15 & 0.752 & 0.658 & 0.743 \\
$\{1,2, \ldots, 16\}$ & 0.15 & 0.763 & 0.675 & 0.759 \\
$\{1,2, \ldots, 17\}$ & 0.15 & 0.775 & 0.690 & 0.765 \\
$\{1,2, \ldots, 18\}$ & 0.15 & 0.789 & 0.703 & 0.779 \\
$\{1,2, \ldots, 19\}$ & 0.15 & 0.797 & 0.715 & 0.790 \\
$\{1,2, \ldots, 20\}$ & 0.15 & 0.809 & 0.725 & 0.803 \\
\hline
\end{tabular}

It can be seen from Table 1, with the change of set $A$, $K=0.05$ before $A$ is $\{1,2,3 \cdots, 5\}, K=0.15$ after $A$ is $\{1,2,3 \cdots, 5\}$, which is always a constant value, this is inconsistent with the facts; The evidence distance $\left(d_{B P A}\right)$, Pignistic probability distance $\left(\operatorname{difBet} P_{m}\right)$ and $\operatorname{ECMC}\left(d_{i j}\right)$ change significantly with the change of the set $\mathrm{A}$, and the change trend of the degree of conflict is basically similar. When the conflict between the evidence becomes smaller, the $d_{B P A}$, difBet $P_{m}$ and $d_{i j}$ can correctly characterize the conflict between the evidence and gradually decrease, for example, the amount of conflict when $A=\{1,2,3\}$ is less than the amount of conflict $A=\{1,2\}$; When $A=\{1,2,3 \cdots, 5\}$, the $d_{B P A}$, $\operatorname{difBet} P_{m}$ and $d_{i j}$ all can represent the minimum degree of conflict; When the conflict between the evidence becomes larger, the 
$d_{B P A}$, difBet $P_{m}$ and $d_{i j}$ can correctly characterize the conflict between the evidence and gradually increased, for example, the amount of collision when $A=\{1,2,3 \cdots, 8\}$ is larger than that at $A=\{1,2,3 \cdots, 7\}$. Through the comparative analysis, the results show that the proposed conflict measure can effectively measure the degree of conflict of evidence.

\section{CONCLUSION}

It is generally considered that the D-S evidence composition theory can not be used when the conflict coefficient $K$ is large. The conflict coefficient $K$ is widely considered as a variable reflecting the conflict between the evidence, but the research shows that the conflict coefficient $K$ can not accurately represent the conflict between the evidence. In this paper, a new variable-ECMC is used to measure the conflict between the evidence, which overcomes the problem that conflict coefficient in classical DS evidence, Jousselme evidence distance and Pignistic probability distance can not be accurate or even unable to characterize the conflict between the evidence. The next step of evidence synthesis can use DS evidence theory and other evidence synthesis method to provide a theoretical basis. In addition, the method proposed in this paper is a closed world conflict measure, without considering the identification framework is incomplete, in the open world, how to measure the conflict between the evidence is still a problem to be solved.

\section{ACKNOWLEDGMENT}

This research is supported by the military scientific research plan, China (No. wj2015cj020001).

\section{REFERENCES}

[1] A. P. Dempster, "Upper and lower probabilities induced by a multivalued mapping," Ann MathematicalStatistics,.vol. 38, pp. 325- 339, 1967.

[2] G. Shafer, A mathematical theory of evidence, Princeton, N J: Princeton $\mathrm{U}$ P,1976.

[3] F. Ye, J. Chen, Y. B. Li, and J. Kang, "Decision-Making Algorithm for Multisensor Fusion Based on Grey Relation and DS Evidence Theory," Journal of Sensors, vol. 2016, Article ID 3954573, 11 pages, 2016.

[4] Y. B. Li, J. Chen, F. Ye, and D. D. Liu, "The Improvement of DS Evidence Theory and Its Application in IR/MMW Target Recognition," Journal of Sensors, vol. 2016, Article ID 1903792, 15 pages, 2016.

[5] Y. X. Zhao, R. F. Jia, and C. Liu, "An Evaluation Method of Underwater Ocean Environment Safety Situation Based on D-S Evidence Theory,” Advances in Meteorology, vol. 2015, Article ID 207656, 8 pages, 2015.

[6] D Bhalla, R K Bansal, and Gupta H O, "Integrating AI based DGA fault diagnosis using dempster-shafer theory," International Journal of Electronical Power \& Energy System, vol. 48, no. 10, pp. 31-38, 2013.

[7] Y. Deng, X. Y. Su, and D. Wang, "Target recognition based on fuzzy dempster data fusion method,” Defence Science Journal, vol. 30,no. 5, pp. 525-530,2010.

[8] J. Fawelt, and V. Myers, "Multiaspect classification of sidescan sonar images: four different approaches to fusing single-aspect information," IEEE Journal of Oceanic Engineering, vol. 35, no. 4, pp. 863-876, 2010.

[9] J. G. Decanini, M. S. Tonelli-Neto, and C. R. Minussi, "Robust fault diagnosis in power distribution systems based on fuzzy ARTMAP neural network-aided evidence theory," IET Generation Transmission \& Distribution, vol. 6,no. 11, pp. 1112-1120, 2012.
[10] R. R. Yager, "On the Dempster-Shafer framework and new combination rules,” Information Science, vol. 41, no. 2, pp. 93-137, 1987.

[11] Q. Sun, X. Q. Ye, and W. K. Gu, "A new combination rules of evidence theory,” Acta Electronica Sinica, vol. 28, no. 8, pp. 117-119, 2000.

[12] E. Lefevre, O. Colot, and P. Vannoorenberghe, "Belief function combination and conflict management," Information Fusion, vol. 3, no. 2, pp. 149-162, 2002.

[13] C. K. Murphy. Combining belief functions when evidence conflicts[J]. Decision Support Systems, 2000, 29(1): 1-9.

[14] H. Luo, Y. P. Yin, and X. X. Hu, "Method to modify evidence source based on trustworthy factors”, Systems Engineering and Electronics, vol. 37,no. 6, pp. 1459-1464, 2015.

[15] W. Jiang, A. Zhang, Y. A. Deng, "Novel Information Fusion Method Based on Our Evidence Conflict Representation," Journal of Northwestern Polytechnical University, vol. 28,no. 1, pp. 27-32, 2010.

[16] O. Basir ,F. Karray , and H. Zhu, “Connectionist based Dempster-Shafer evidential reasoning for data fusion,” IEEE Trans. on Neural Networks, vol. 16,no. 6, pp. 1513-1530, 2005.

[17] C. H. Hu, and X. S. Si, "An improved D-S algorithm under the new measure criteria of evidence conflict," Acta Electronica Sinica, vol. 37,no. 7, pp. 1578-1583, 2009.

[18] X. Y. Su, M. Sankaran, and P. D. Xu, "Handling of dependence in Dempstet-Stealer theory," International Journal of Intelligent Systems,vol. 30, no. 4, pp. 441-467, 2015.

[19] W. R. Liu, "Analyzing the degree of conflict among belief functions," Artificial Intelligence, vol. 170,no. 11, pp. 909-924, 2006.

[20] Y. Deng, D. Wang, and Q. Li, “A new method to analyze evidence conflict,” Control Theory \& Application, vol. 28, no. 6, pp. 839-844, 2011.

[21] A. L. Jousselme, D. Grenier, and E. Bosse, "A new distance between two bodies of evidence," Information Fusion, vol. 2, no. 2, pp. 91$101,2001$.

[22] W. Jiang, and J. Y. Peng, "New representation method of evidential conflict," Systems Engineering and Electronics, vol. 32, no. 3, pp. 562565, 2010.

[23] Z. G. Liu, and Y. M. Cheng, "Combination of weighted belief functions based on evidence distance and conflicting belief," Control Theory \& Applications, vol. 26, no. 12, pp. 1439-1442, 2009.

[24] W. H. Bi, A. Zhang, and C. Li, "Weighted evidence combination method based on new evidence conflict measurement approach,” Control and Decision, vol. 31, no. 1, pp. 74-78, 2016.

[25] C. X. Li, Y. Zhou, and S. C. Wang, "A Novel Combination Rule of Evidence Theory in Multi-Source Information Fusion,” Journal of shanghai jiao tong university, vol. 50, no. 7, pp. 1125-1131, 2016. 